\title{
Preliminary evaluation of the performance of a new, highly sensitive commercial immunoassay for serum ferritin determination
}

\author{
Paolo Signò ${ }^{1}$, Alessandra Barassi ${ }^{2, *}$, Raffaele \\ Novario ${ }^{3}$ and Gian Vico Melzi d'Eril ${ }^{4}$ \\ ${ }^{1}$ Laboratorio di Analisi, Ospedale di Circolo, Varese, \\ Italy \\ ${ }^{2}$ Dipartimento di Scienze Biomediche Sperimentali e \\ Cliniche, Università degli Studi dell'Insubria, Varese, \\ Italy \\ ${ }^{3}$ Fisica Sanitaria, Ospedale di Circolo, Varese, Italy \\ ${ }^{4}$ Dipartimento di Medicina, Chirurgia e Odontoiatria, \\ Università degli Studi di Milano, Milano, Italy
}

\begin{abstract}
We evaluated the analytical performance of a new, commercial, fully automated immunoturbidimetric assay for the determination of ferritin [FERLatex(X2)CN SEIKEN, Denka Seiken, Japan] in serum on the Olympus AU2700 analyzer. The new assay is a latex-enhanced turbidimetric immunoassay with an analysis time of $10 \mathrm{~min}$. The linearity of the assay was confirmed up to $2505 \mathrm{pmol} / \mathrm{L}\left(\mathrm{R}^{2}=0.999\right)$. The detection limit and the functional sensitivity were both $4.5 \mathrm{pmol} / \mathrm{L}$. The intra- and inter-assay imprecision (CV) at $67,506,2186 \mathrm{pmol} / \mathrm{L}$ was $<1.8 \%$ and $<2.5 \%$, respectively. Verification of the traceability to a $\mathrm{WHO}$ standard $(80 / 578)$ showed a recovery of $102.6 \%$ (target value $449 \mathrm{pmol} / \mathrm{L}$ ). No hook effect was observed in samples containing up to $33,705 \mathrm{pmol} / \mathrm{L}$. The assay showed good correlation with the Beckman Immage nephelometric system $(r=0.999)$. Hemoglobin $(\leq 9.8 \mathrm{~g} / \mathrm{L})$, total bilirubin $(\leq 113 \mu \mathrm{mol} / \mathrm{L})$, conjugated bilirubin $(\leq 109 \mu \mathrm{mol} / \mathrm{L})$ and rheumatoid factor $\left(\leq 5.2 \times 10^{5} \mathrm{IU} / \mathrm{L}\right)$ did not interfere with the assay. The reference interval (2.5-97.5 percentile) was 72-521 $\mathrm{pmol} / \mathrm{L}$ for men and $27-267 \mathrm{pmol} / \mathrm{L}$ for women. The reference interval in patients with anemia, malignant tumors and hemochromatosis was 5.6-52, 130-2436 and $1465-2903 \mathrm{pmol} / \mathrm{L}$, respectively. On the basis of the receiver operating characteristic curve, the $90 \%$ sensitivity cut-off value to distinguish between patients with and without iron deficiency was $40 \mathrm{pmol} / \mathrm{L}$. The new latex turbidimetric procedure for ferritin assay is an attractive alternative that avoids the need for dedicated instrumentation.
\end{abstract}

Keywords: automation; ferritin; turbidimetric immunoassay.

*Corresponding author: Alessandra Barassi, via Monte Rosa 35, 21020 Buguggiate, Varese, Italy

Phone: +39-03355444121, E-mail: alessandra.barassi@tin.it
Ferritin is a large, multifunctional, multisubunit protein that plays an important role in iron storage and metabolism. Its ability to sequester the element gives ferritin the double function of iron detoxification and reserve (1). Ferritin assays must have a broad dynamic range, as the serum concentrations can vary from $>2247 \mathrm{pmol} / \mathrm{L}$ in some types of malignancies (2) to $<23 \mathrm{pmol} / \mathrm{L}$ in iron-deficiency anemia (3). The determination of serum ferritin can be useful in detecting hemochromatosis in most subjects in a precirrhotic stage without irreversible complications (4). Finally, preliminary studies suggest that increased ferritin might be an independent predictor of premature coronary artery disease in male patients (5). Thus, precise measurement in the lower range is required for the diagnosis of iron-deficiency anemia, while a wide analytical range is useful for measurement in patients with tumors, inflammation, hemochromatosis or atherosclerosis.

The aim of this study was to evaluate a new serum ferritin assay, FER-Latex(X2)CN SEIKEN (Denka Seiken, Tokyo, Japan), on the Olympus AU 2700 analyzer (Olympus Diagnostics GmbH, Hamburg, Germany) by defining key performance characteristics, including detection limit, linearity on dilution, recovery, imprecision, comparison with a nephelometric method (Beckman Immage Assay, Beckman-Coulter, Fullerton, CA, USA), reference interval and cut-off value for iron-deficiency anemia. The method chosen for comparison is a well-established and widespread system for ferritin determination and the standards were calibrated against the 80/578 WHO Reference Preparation. The new assay is a latex-enhanced turbidimetric immunoassay that uses six different calibrators. Calibrator $A$ is a $10 \mathrm{mmol} / \mathrm{L}$ HEPES buffer $(\mathrm{pH} \mathrm{7.5)}$. Calibrators B-F contain increasing concentrations of ferritin (67, 225, 450, 1124 and 2247 pmol/L) in HEPES buffer. These calibrators are traceable to the WHO standard (80/602).

The new FER-Latex method on the Olympus analyzer requires $15 \mu \mathrm{L}$ of sample, $180 \mu \mathrm{L}$ of Reagent 1 (170 mmol/L glycine buffer, $\mathrm{pH} 8.3$ ) and $80 \mu \mathrm{L}$ of Reagent 2. Reagent 2 consists of a suspension of polystyrene latex particles in $170 \mathrm{mmol} / \mathrm{L}$ glycine buffer (pH 7.3) coated with anti-human ferritin polyclonal antibodies. The latex particles are agglutinated according to the ferritin dose, which causes increased turbidity that is detected at $660 \mathrm{~nm}$, with a total analysis time of $10 \mathrm{~min}$. For comparison, the Beckman Immage assay was performed according to the manufacturer's recommendations. 


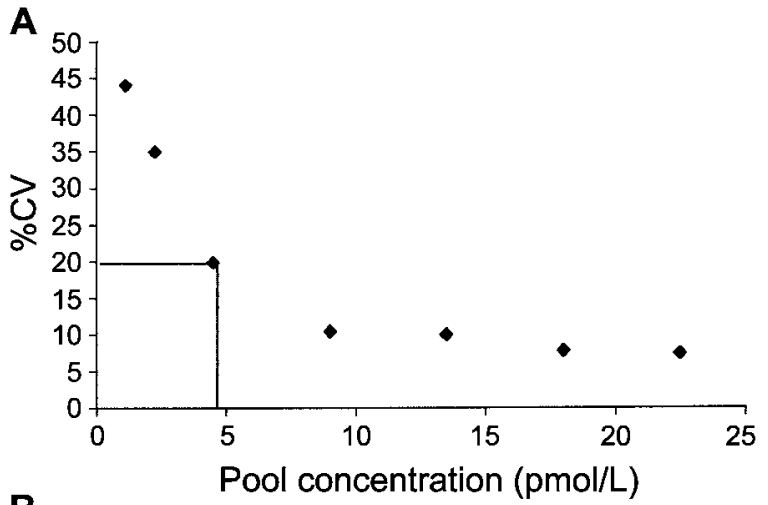

B

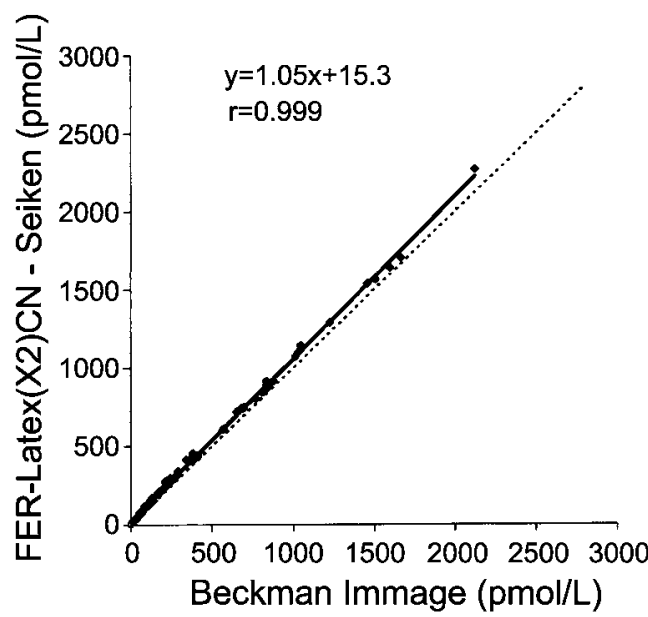

Figure 1 Functional sensitivity (A) defined as the lowest measurable ferritin concentration with a CV of $<20 \%$. Comparison of the FER-Latex(X2)CN SEIKEN assay with the Beckman Immage assay (B) according to Passing-Bablok regression analysis. The dashed line represents the equivalence line.

We examined the linearity of the new method by analyzing 10 protein concentrations. Fresh plasma samples were used to prepare a high-concentration patient plasma pool ( $>2247 \mathrm{pmol} / \mathrm{L})$. The undiluted pool and nine separate dilutions (9:1, 8:2, 7:3, 6:4, 5:5, $4: 6,3: 7,2: 8$, and $1: 9$ ) were assayed in duplicate in the same analytical run. Linear regression analysis was carried out and Pearson's correlation coefficient was calculated $\left(y=0.988 x-5.512 ; R^{2}=0.999\right)$. The results (percentage of theoretical concentrations) showed values between $96.2 \%$ and $98.6 \%$, with a median bias of $-2.8 \%$; therefore, the method is linear within its measurement range $(2505 \mathrm{pmol} / \mathrm{L}$ was the mean of the high-concentration pool). The standard error of regression $\left(\mathrm{S}_{\mathrm{y} / \mathrm{x}}\right)$ and t-test for the regression analysis were 3.082 and $343.4(p<0.001)$, respectively. The minimum detectable ferritin concentration was assessed by 21 replicate measurements of the zero calibrator in a single run and defined as the ferritin value corresponding to a signal 2 SD greater than the mean found for this sample. On the basis of this procedure, the detection limit was $4.5 \mathrm{pmol} / \mathrm{L}$ for the assay. In addition, the functional sensitivity, defined as the concentration with an inter-assay imprecision (CV) $<20 \%$, was $4.5 \mathrm{pmol} / \mathrm{L}$ (Figure $1 \mathrm{~A}$ ). This coincidence of the functional sensitivity with the detection limit is probably a consequence of the reagents (buffers), which are unaffected by the matrix. This study was performed by preparing seven serial dilutions of a ferritin pool, ranging from 1.1 to $23 \mathrm{pmol} / \mathrm{L}$. To determine between-run imprecision, three pooled patient serum samples were aliquoted and frozen at $-20^{\circ} \mathrm{C}$. Two replicates/specimens were analyzed per run and one run per day for 21 days, using two reagent lots and two calibrations. CVs calculated from the means of each run were $2.5 \%$ (mean concentration $70 \mathrm{pmol} / \mathrm{L}$ ), 1.5\% (mean concentration 510 $\mathrm{pmol} / \mathrm{L}$ ) and 2.0\% (mean concentration $2209 \mathrm{pmol} / \mathrm{L}$ ). Within-run $\mathrm{CVs}$ were $1.8 \%$ (mean concentration $67 \mathrm{pmol} / \mathrm{L}$ ), 0.5\% (mean concentration $497 \mathrm{pmol} / \mathrm{L}$ ) and $1.2 \%$ (mean concentration $2164 \mathrm{pmol} / \mathrm{L}$ ). In this study, 21 replicates were analyzed in duplicate in the same analytical run.

The recovery of controls (Immuno-Q-sera II SEIKEN) calculated by the difference between expected and observed values (percentage recovery) was $98.1 \%$ $(\mathrm{n}=15$; target value $243 \mathrm{pmol} / \mathrm{L}$, mean $\pm \mathrm{SD}=$ $238 \pm 4.3 \mathrm{pmol} / \mathrm{L})$ and $99.3 \%(\mathrm{n}=15 ;$ target value $944 \mathrm{pmol} / \mathrm{L}$, mean $\pm \mathrm{SD}=937 \pm 9.7 \mathrm{pmol} / \mathrm{L})$. Additional recovery experiments using two different concentrations of the calibrator in five samples gave a mean recovery from ten measurements of $98.8 \%$ (range 97.3-101.5\%) of the calculated value. Verification of the traceability to the WHO standard, using the Beckman-Coulter Calibrator for ferritin $(80 / 578$ WHO standard), showed a recovery of $102.6 \% \quad(n=15$; target value $449 \mathrm{pmol} / \mathrm{L}$, mean $\pm \mathrm{SD}=461 \pm 4.0 \mathrm{pmol} / \mathrm{L}$ ). To determine whether highly increased concentrations of ferritin cause a "hook effect", a high-concentration serum pool was diluted with calibrator A. Final concentrations ranged from 2022 to $53,928 \mathrm{pmol} / \mathrm{L}$ and the samples were analyzed in duplicate. The results obtained from the undiluted samples were compared with those obtained from the same samples diluted to fall within the range of linearity. No hook effect was observed in samples containing up to $33,705 \mathrm{pmol} / \mathrm{L}$ of antigen, because the difference between the two determinations was below 5\%. Passing and Bablok regression analysis (6) was used for method comparison between the FER-Latex(X2)CN SEIKEN assay and the Beckman Immage nephelometric system. To avoid any dilution, we limited the range for this experiment to the ranges reported for the two ferritin methods. For the comparison study, we used samples from 120 patients scheduled for ferritin determination for diagnostic purposes and/or monitoring of medical treatment. Passing and Bablok regression analysis (Figure 1B) revealed an intercept of $15.3 \mathrm{pmol} / \mathrm{L}$ (95\% Cl 13-20), a slope of 1.05 (95\% Cl 1.04-1.06), and a coefficient of correlation ( $r$ ) of 0.999 , suggesting that the values obtained with the new method are slightly higher than those obtained with the comparison assay. As a consequence the [(compared)(comparison)] mean differences at the lower and higher reference limits for males and females $(72,27$, 521 and $267 \mathrm{pmol} / \mathrm{L})$ are 16, 17, 40 and $27 \mathrm{pmol} / \mathrm{L}$, respectively.

Using a pool of samples with a mean concentration of $211 \mathrm{pmol} / \mathrm{L}$ of ferritin spiked with interferents and processed in duplicate did not affect results significantly, even though the concentrations of interferents 
in spiked samples were $113 \mu \mathrm{mol} / \mathrm{L}$ total bilirubin (recovery 100.0\%), $109 \mu \mathrm{mol} / \mathrm{L}$ conjugated bilirubin (recovery 101.0\%), $9.8 \mathrm{~g} / \mathrm{L}$ hemoglobin (recovery $103.0 \%$ ) and $5.2 \times 10^{5} \mathrm{IU} / \mathrm{L}$ rheumatoid factor (recovery $102.8 \%)$. The spiking solutions were prepared from stock solutions containing known levels of interferents. Stored calibration curves were compared with fresh calibration curves. Two levels of controls and two human serum pools were analyzed at 1-week intervals. The calibration curve is stable for at least 1 month (error $<5 \%$ ).

Four groups of subjects entered the clinical evaluation study. Group 1 included 140 (70 males and 70 females; median age 39 years; range 21-53 years) healthy individuals using exclusion criteria as suggested in IFCC recommendations on the theory of reference values (7). The other three groups consisted of 80 iron-deficiency anemia patients, 80 subjects with a malignant tumor and 25 hemochromatosis patients. The three pathological groups were classified according to the following criteria: transferrin saturation $<15 \%$ and hemoglobin $<140 \mathrm{~g} / \mathrm{L}$ in males or $<120$ $\mathrm{g} / \mathrm{L}$ in females for iron-deficiency anemia patients; histolopathological findings for subjects with a malignant tumor; and homozygosity for the C282Y mutation of the HFE gene for patients with hemochromatosis. The procedures followed were in accordance with the current revision of the Helsinki Declaration (8). The reference interval for ferritin calculated by non-parametric determination of 2.5-97.5 percentiles was 72-521 pmol/L for men and 27-267 pmol/L for women. The reference interval in patients with anemia, malignant tumors and hemochromatosis was 5.6-52, 130-2436 and 1465-2903 $\mathrm{pmol} / \mathrm{L}$, respectively.

To determine the diagnostic accuracy of the assay for anemia, we performed receiver operating characteristic $(\mathrm{ROC})$ plot analysis, and the area under the curve (AUC) was calculated. The cut-off value was determined at the $90 \%$ sensitivity criterion derived directly from the ROC curve. All probabilities were two-tailed, and $p$-values $<0.05$ were regarded as statistically significant. When distinguishing between patients with and without anemia $(n=80$ and $n=100$, respectively) the AUC (SE) was $0.967(0.013)$ using the FER-Latex(X2)CN SEIKEN assay $(95 \% \mathrm{Cl}$, 0.9290.988). The AUC (SE) was comparable using the Beckman Immage assay: $0.979(0.015)(95 \% \mathrm{Cl}, 0.926-$ $0.990)$. On the basis of the ROC curve, $40 \mathrm{pmol} / \mathrm{L}$ was the cut-off value for anaemia at $90 \%$ sensitivity $(95 \%$ $\mathrm{Cl}, 81.2-95.6)$ and $92 \%$ specificity $(95 \% \mathrm{Cl}, 84.8-96.5)$ for the FER-Latex(X2)CN SEIKEN assay. Using the Beckman Immage assay, the cut-off value was $36 \mathrm{pmol} / \mathrm{L}$ at $90 \%$ sensitivity $(95 \% \mathrm{Cl}, 82.4-96.1)$ and 91\% specificity (95\% Cl, 83.9-94.5).
In conclusion, the method evaluated here can be operated on several common automated clinical chemistry analyzers; the new assay offers a high degree of reproducibility, together with an extended range, allowing direct measurement of the majority of the samples without further external sample dilution. No statistically significant bias in linearity was observed when samples were diluted. An analytical and functional sensitivity of $4.5 \mathrm{pmol} / \mathrm{L}$ and imprecision of $\mathrm{CV}<3 \%$ in all cases make the assay suitable for use in the diagnosis and monitoring of iron-deficiency anaemia. The wide analytical measurement range $(4.5-2471 \mathrm{pmol} / \mathrm{L})$ is useful for patients with tumors, inflammation or hemochromatosis. No substantial differences were observed by comparing results of the new turbidimetric assay with the Beckman nephelometric assay. In addition, the calibration curve shows good stability (1 month).

The new latex turbidimetric procedure for ferritin assay is an attractive alternative that avoids the need for dedicated instrumentation. Finally, this is an example of instrument consolidation without a deterioration in quality performance, an important goal for every clinical laboratory.

\section{References}

1. Harrison PM, Arosio P. The ferritins: molecular properties, iron storage function and cellular regulation. Biochim Biophys Acta 1996;1275:161-203.

2. Ockhulzen T, Kok AJ, Sleijfer DT, de Bruijn HWA, Schraffordt-Koops $H$, Marrink J. Serum ferritin determinations are of no value in the management of patients with disseminated non-seminomatous germ cell tumors. Eur $\mathrm{J}$ Cancer Clin Oncol 1985;21:931-4.

3. Limet JN, Collet-Cassart D, Magnusson CG, Sauvage P, Cambiaso CL, Masson PL. Particle counting immunoassay (PACIA) of ferritin. J Clin Chem Clin Biochem 1982; 20:141-6.

4. Niederau C, Strohmeyer G. Strategies for early diagnosis of haemochromatosis. Eur J Gastroenterol Hepatol 2002; 14:223-9.

5. Haidari M, Javad Ei, Sanati A, Hajilooi M, Ghanbili J. Association of increased ferritin with premature coronary stenosis in men. Clin Chem 2001;47:1666-72.

6. Passing $\mathrm{H}$, Bablok W. A new biometrical procedure for testing the equality of measurements from two different analytical methods. Application of linear regression procedures for method comparison studies in clinical chemistry. Part I. J Clin Chem Clin Biochem 1983;21:709-20.

7. PetitClerc $C$, Solberg HE. IFCC approved recommendation (1987) on the theory of reference values. Part 2. Selection of individuals for the production of reference values. J Clin Chem Clin Biochem 1987;25:639-44.

8. World Medical Association. Declaration of Helsinki: ethical principles for medical research involving human subjects. J Am Med Assoc 2000;284:3043-5.

Received February 14, 2005, accepted May 17, 2005 